\title{
MODEM: a multi-agent hierarchical structure to model the human motor control system
}

\author{
Mehran Emadi Andani · Fariba Bahrami • \\ Parviz Jabehdar Maralani · Auke Jan Ijspeert
}

Received: 15 March 2008 / Accepted: 2 October 2009

(c) Springer-Verlag 2009

\begin{abstract}
In this study, based on behavioral and neurophysiological facts, a new hierarchical multi-agent architecture is proposed to model the human motor control system. Performance of the proposed structure is investigated by simulating the control of sit to stand movement. To develop the model, concepts of mixture of experts, modular structure, and some aspects of equilibrium point hypothesis were brought together. We have called this architecture MODularized Experts Model (MODEM). Human motor system is modeled at the joint torque level and the role of the muscles has been embedded in the function of the joint compliance characteristics. The input to the motor system, i.e., the central command, is the reciprocal command. At the lower level, there are several experts to generate the central command to control the task according to the details of the movement. The number of experts depends on the task to be performed. At the higher level, a "gate selector" block selects the suitable subordinate expert considering the context of the task. Each expert consists of a main controller and a predictor as well as
\end{abstract}

M. Emadi Andani ( $\varangle)$

Department of Biomedical Engineering, Faculty of Engineering,

University of Isfahan, Isfahan, Iran

e-mail: Emadi@eng.ui.ac.ir

M. Emadi Andani · F. Bahrami · P. Jabehdar Maralani Control and Intelligent Processing Center of Excellence, CIPCE, University of Tehran, Tehran, Iran

F. Bahrami

e-mail: fbahrami@ut.ac.ir

P. Jabehdar Maralani

e-mail: pjabedar@ut.ac.ir

M. Emadi Andani · A. J. Ijspeert

Biologically Inspired Robotics Group (BIRG), School of Computer and Communication Sciences, EPFL, Lausanne, Switzerland

e-mail: auke.ijspeert@epfl.ch several auxiliary modules. The main controller of an expert learns to control the performance of a given task by generating appropriate central commands under given conditions and/or constraints. The auxiliary modules of this expert learn to scrutinize the generated central command by the main controller. Auxiliary modules increase their intervention to correct the central command if the movement error is increased due to an external disturbance. Each auxiliary module acts autonomously and can be interpreted as an agent. Each agent is responsible for one joint and, therefore, the number of the agents of each expert is equal to the number of joints. Our results indicate that this architecture is robust against external disturbances, signal-dependent noise in sensory information, and changes in the environment. We also discuss the neurophysiological and behavioral basis of the proposed model (MODEM).

Keywords Human motor control - Mixture of experts . Modular structure - Multi-agent · Hierarchical structure . Equilibrium point hypothesis $\cdot$ Sit to stand

\section{Introduction}

How the central nervous system (CNS) determines the motor commands to make a movement is one of the main questions of human movement studies.

In one possible modeling approach, principles of optimal control are used to answer this question. Although modeling approaches based on optimal control (Harris and Wolpert (1998) and Todorov and Jordan (2002)) are in general able to explain different aspects of human motor control, they are in our opinion computationally too demanding to describe the computations performed by the CNS. Indeed, any given optimal controller must repeat the whole computational 
procedure whenever there are uncertainties (like unpredicted external disturbances) in the system (here: in the motor system). As Cisek (2005) mentioned "a plan which optimizes nearly every aspect of movement" places heavy computational burden on the CNS. This fact seems to us to contradict the principle of minimal interaction proposed by Balasubramaniam and Feldman (2004) which states that the motor control system has a natural tendency to minimize the overall activity and interactions in both the CNS and the musculoskeletal system. From this stand point, a suitable model to describe human motor control system should be less computationally demanding.

In this article, we are therefore looking for models to describe the motor control system which are computationally less demanding and at the same time interesting features such as motor memory, the capability to use different types of learning, e.g., learning from trial and error or imitation learning, generalization, knowledge of physical properties of the body and the environment. The notion of dynamical internal models is well suited to provide these interesting features. In this point of view, which is inspired from the field of robotics, it is proposed that the brain learns to construct internal models of the motor system and the environment in interaction with it. There are two groups of internal models; inverse and forward dynamics models (Gomi and Kawato 1993; Wolpert and Kawato 1998; Doya et al. 2002). According to another point of view, some more sophisticated models were developed based on both inverse and forward internal models with a modular structure (Gomi and Kawato 1993; Jordan and Jacobs 1994; Wolpert and Kawato 1998; Haruno et al. 2001; Doya et al. 2002; Haruno et al. 2003). Those models are in agreement with the distributed and hierarchical nature of the motor control system and they are computationally less demanding than simple forward or inverse internal models. A famous architecture proposed by Haruno et al. (2001) is the Modular Selection and Identification for Control (MOSAIC) for parallel-distributed switching. MOSAIC is a hierarchical motor control structure to describe human motor learning. The original idea of this study was proposed previously by several researchers (Wolpert et al. 1998; Wolpert and Kawato 1998; Wolpert and Ghahramani 2000). This model was developed for motor control under multiple different contexts and consists of several modules. Each module includes a forward model, named the predictor, and an inverse model, called the controller (Haruno et al. 2001). This structure simultaneously learns multiple internal inverse models to control the task, and at the same time it learns to select the suitable inverse models for a specified environment. The inverse model calculates a motor command, while the forward model predicts the outcome of the current command. The accuracy of the prediction of the forward model of each module is represented by the responsibility signal of the module. The responsibility signals were applied to weight the outputs of each inverse model. These signals were also used for competitive learning of the forward and inverse models (Haruno et al. 2001; Doya 2002).

Another modular structure proposed for motor control is the mixture of experts (MEX) (Gomi and Kawato 1993; Jordan and Jacobs 1994; Graybiel et al. 1994). In the MEX, each expert is a function approximator. Outputs of the experts are linearly combined by a classifier (gating module) that plays the role of a centralized switch. Each expert model is trained using a subpart of the input data corresponding to conditions and constraints that it must become specialized in. The gating module determines the degree of contribution of each expert in generating the final output. Therefore, the selection (switching activity) is centralized in the gating module and is independent of the activity of the expert modules.

In comparison to the MEX model, internal models of the MOSAIC are experts for the controlled objects (here in the field of motor control, controlled object is a given movement) and contribute significantly in the switching activity (Imamizu et al. 2004). In the MOSAIC model, multiple pairs of forward and inverse models are coupled. For example, when we use a new pen to write, forward models (Blakemore et al. 1998; Iacoboni 2001; Miall et al. 2001) of different types of pens simultaneously predict sensory feedback using an efference copy of motor commands. Then, the prediction of each forward model is compared with actual feedback. A forward model with the smallest error indicates that particular forward model must have been the proper predictor in the current context. Then, the inverse model paired with that suitable predictor is considered as the suitable controller. In this way, the selection mechanism, which is a soft selection, depends on the internal models.

One can generate a given movement under different conditions and contexts. For example, the main features of the kinematics of the movement during writing are conserved, while the pen and its effect on the dynamics of the system are different. This special case alludes to a more general conclusion, that in higher levels of human motor control system, there is most probably a unique representation for any given movement, and when the movement is executed, variations and uncertainties in the dynamics of the motor system are compensated at lower levels of motor control system. Therefore, according to different observations and neurophysiological facts, we may conclude that an appropriate model to describe the human motor control system has a hierarchical, distributed, and modular structures (Pocock and Richards 1999; Guyton and Hall 2006).

As an alternative to internal models, equilibrium point hypothesis (EPH) is another theoretical framework employed in human motor control field (Feldman and Latash 2005; Gu and Ballard 2006). According to the theory of EPH, movement is controlled by shifting muscle activation thresholds. In other words, limb postures are interpreted as equilibrium 
points ( $\mathrm{Gu}$ and Ballard 2006) and a movement is generated by transitions from one body posture to another by resetting control parameters of the motor system (Balasubramaniam and Feldman 2004). In this case, the previous position is a deviation from newly readdressed position, and the forces are generated to transfer a joint to its new equilibrium. One of the important notions used by EPH is Joint Compliant Characteristics (JCC) (Domen et al. 1999). Slope and intercept of JCC, defined as $C$ and $R$, are two main regulatory parameters (variables) that CNS uses to control the joint compliant and through it equilibrium point of the limb. In fact, JCC and the external force field together determine the equilibrium point of a limb. The parameter $R$ is interpreted as the equilibrium position of the joint in the absence of the external force. According to the physiological notions, it corresponds to the reciprocal activation of the antagonist muscles of the joint. Command $C$ based on the same point of view, represents the co-activations of the antagonist muscles. To accomplish a given movement, the JCC changes along with the external forces. This will need changes in $R$ and $C$ parameters during the movement. Therefore, $R$ and $C$ can be considered as control parameters determining dynamic sequence of equilibrium positions.

As Imamizu et al. (2004) have discussed centralized and distributed switching are two strategies applied by the CNS to control motor tasks. Distributed switching strategy can be expressed by modular structures like MOSAIC, and centralized switching can be modeled by structures like MEX. Therefore, we proposed a new architecture based on the concepts of modular architecture, including both centralizedand decentralized-distributed switching strategies together with the concept of the reciprocal command deduced from EPH. We named this model MODularized Experts Model (MODEM).

The architecture of MODEM is developed based on the fact that the cerebellum and basal ganglia create distinct closed loop circuits with different regions of the cerebral cortex including the frontal, premotor, primary motor, and parietal cortices (Middleton and Strick 1998). MODEM has a hierarchical structure (two main levels) and at the lower level it is consisted of several experts. A "gate selector" block is in the highest level to select which subordinate experts should attempt to control the task at the ongoing situation. In the proposed architecture, each expert consists of a main controller and several auxiliary modules. Each auxiliary module is in fact an agent which cooperates in controlling the task performance if the main controller fails to do it accurately. An agent in each expert is responsible for correcting the movement of one joint. Each agent learns to reject a simple constant disturbance in its corresponding joint and then generalizes this learning to a more complicated situation (different disturbances). The output of MODEM, i.e., the central command that is sent to the motor system, is the reciprocal command.
Thereby, in accordance with neuromuscular properties, the role of the reciprocal command in generating joint torques is integrated into the architecture of MODEM. An important advantage of this feature is that the intrinsic feedback in muscles (due to the structure of JCC) will increase the stability of the model (Partridge 1967). In this study, it is assumed that the co-activation command is constant.

The performance of the proposed structure is investigated by simulating the control of sit-to-stand (STS) transfer from a chair. Our results show that the MODEM architecture can robustly learn and control the movement. According to our simulation results, the proposed architecture controls robustly the movement in the presence of additional noise in sensory feedback, external disturbance, and changes in the dynamics of the environment.

The article is organized as follows: in Sect. 2, the MODEM architecture is introduced. We explain how the controllers must be trained by a sequential procedure. Then, in Sect. 3, the simulation experiments are presented. In Sect. 4, we discuss possible evidences justifying correspondences between the MODEM architecture and behavioral observations as well as neurophysiological facts. Finally, in Sect. 5 concludes the article.

\section{Introducing the architecture of MODEM}

Based on different observations, one may distinguish between two separate processes in human motor control system: motor planning and motor execution (Nakano et al. 1999; Cisek 2005). At the level of motor planning, the CNS plans a desired movement in accordance with the type of task to be performed and the corresponding physical and physiological constraints. Then at the level of execution, the CNS generates appropriate central motor commands so that the motor system may track the desired trajectory appropriately. At this level, the CNS functions as a controller.

The proposed structure for motor execution level and its relation to the motor system are shown in Fig. 1A. In the proposed structure, the motor control system consists of the following functional blocks: the MODEM, the forward model of motor system and the feedback controller. the structure of MODEM will be explained in more detail later. The model of motor system consists of two blocks: the JCC block and the model of body-skeletal dynamics. The JCC block is defined in Sect. 2.1 using (1) and the Model of body-skeletal dynamics for the case study used in this article will be explained in Sect. 3.2. The "forward model of motor system" is similar to "the model of motor system". The feedback controller is a proportional controller that will be explained in more detail in Sect. 3.4.

The local feedback loop, which consists of the forward model of the motor system and the feedback controller, is in 


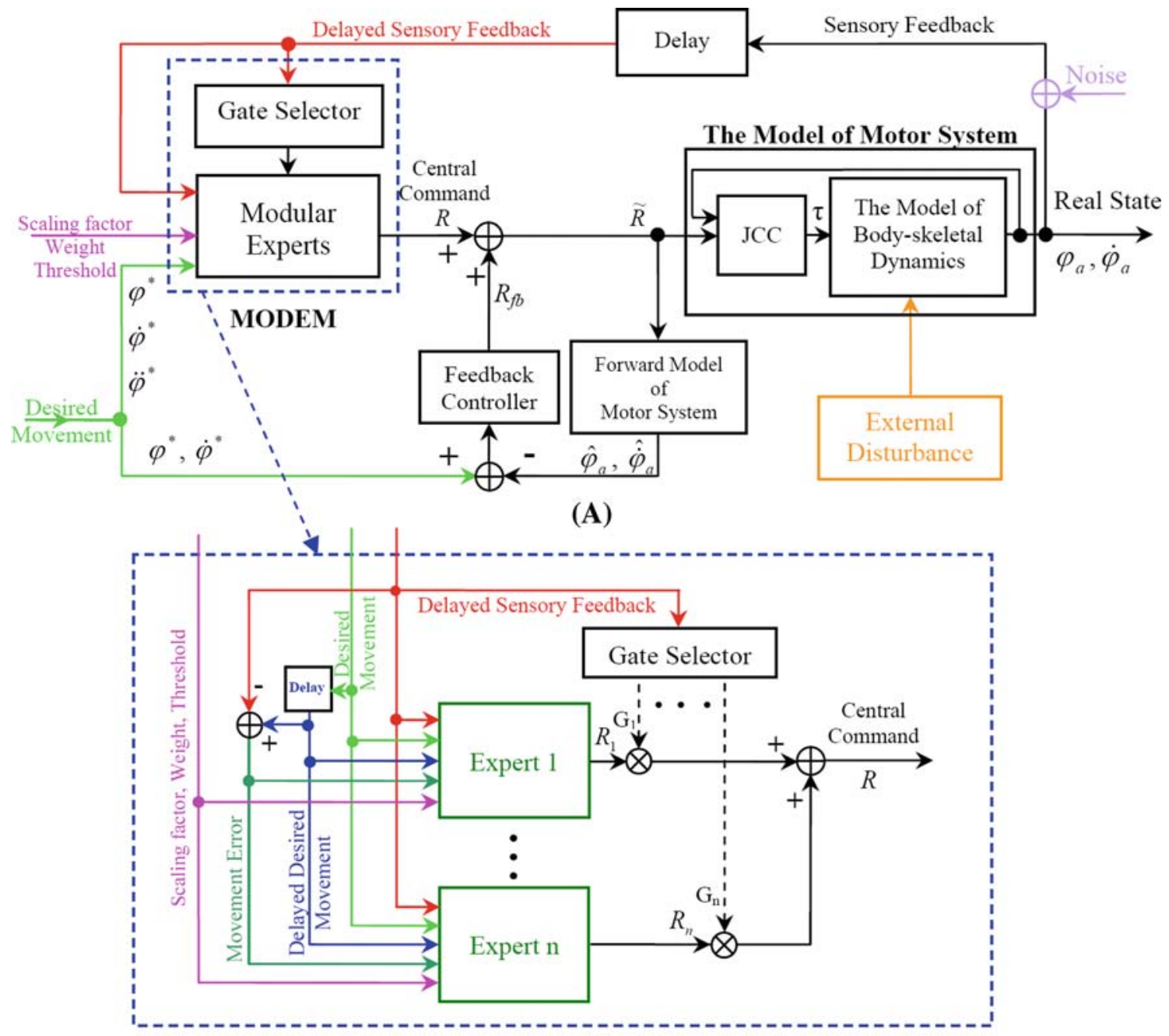

(B)

Fig. 1 A Schematic of whole system including the MODEM. The motor control system is including the MODEM, the Forward Model of Motor System and the Feedback Controller; the Feedback Controller is described by Eq. 9 . The central command $(\tilde{R})$ which is generated by motor controller in interaction with the motor system, is transformed into the joint torque by the JCC block (described by (1)). The noise

fact the inner loop of a Smith predictor structure. The forward model predicts the results of the actual central motor command. The prediction outcome is compared with the desired movement and the resulted error is used to improve the command $(R)$ generated by the MODEM.

In addition, the noise in the sensory information and the external disturbance in the motor system are modeled by an additional noise input signal and an "External Disturbance" block in Fig. 1A, respectively.

\subsection{Central motor commands and joint torques}

In the structure proposed by Haruno et al. (2001) for motor execution level, there are two groups of controllers: the so- in the sensory information is modeled by an additional noise block. Furthermore, the External Disturbance block models the external disturbances applied to the motor system (at different joints) during task performance. B Schematic of the MODEM. The MODEM is described by (5)

called MOSAIC, and a feedback controller (a proportional controller). The MOSAIC controller uses a combined policy, i.e., it applies feedforward and feedback information to generate central motor commands. In this study, we propose a new structure for the motor execution level (see Fig. 1A). The combined controller in this case consists of a set of multiple experts (see Fig. 1B). Each expert has in turn a modular structure (see Fig. 2). That is why we called this model MODEM.

The central command $(\tilde{R})$ is generated in joint angle space by the motor controller and in interaction with the motor system and the environment. This is one of the main differences between the MODEM and the MOSAIC models. Central commands in MOSAIC are muscle force or joint torque. According to Feldman and Latash (2005), the reciprocal 


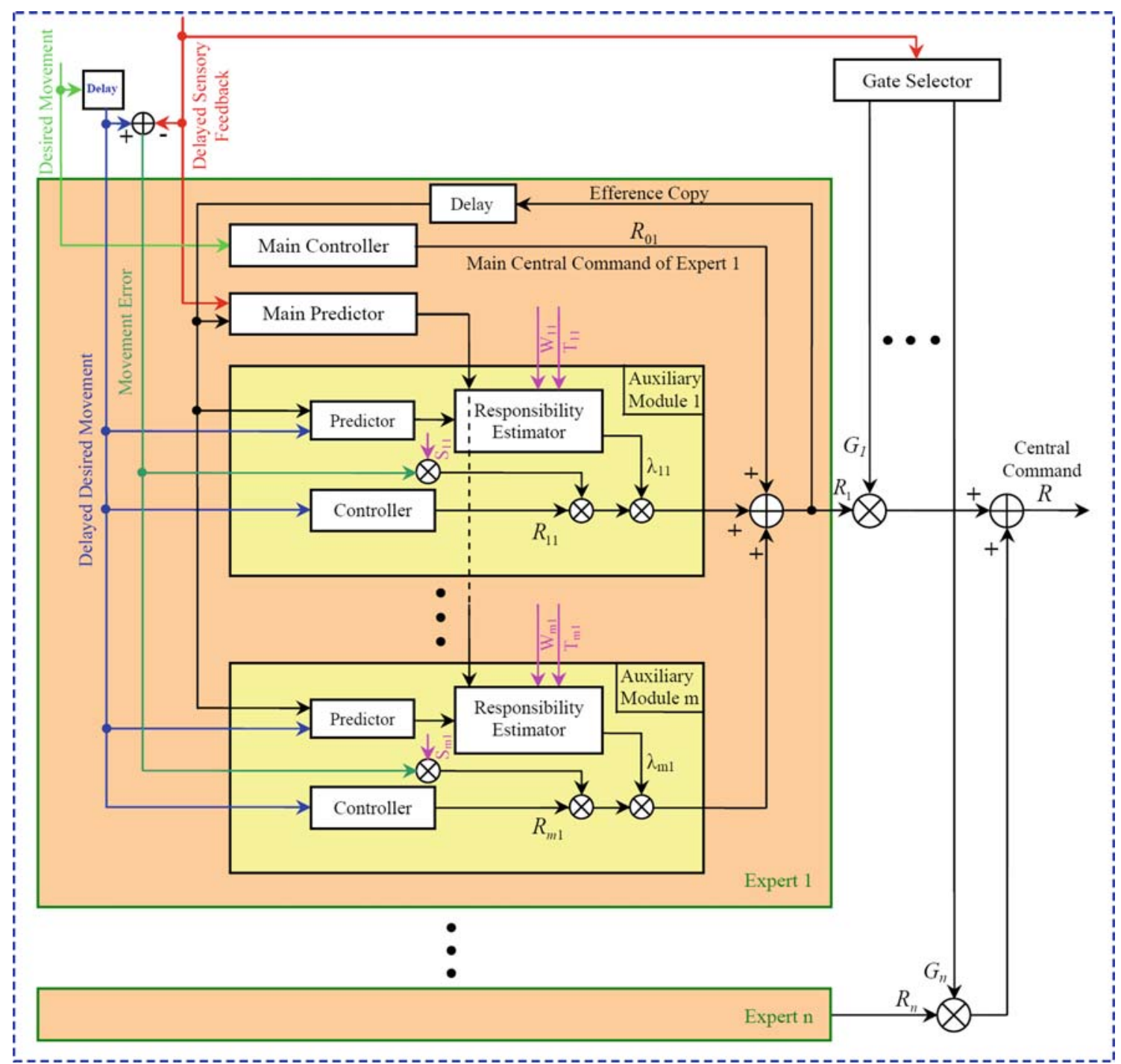

Fig. 2 Schematic of the MODEM. Details of an expert including the main controller (described by linear network), main predictor (described by (2)) and the auxiliary modules are shown. Each auxiliary module is consisted of a controller (described by linear network), a predictor

command can generate appropriate joint torques considering the simplified joint stiffness relation (represented in Fig. 1A by JCC block):

$\tau_{i}=K_{i}\left(\varphi_{a, i}-R^{i}\right)$

where $\tau_{i}$ is the torque of the $i$ th joint, $R^{i}$ is the reciprocal command for the $i$ th joint and is the $i$ th element of the final central command $R \cdot \varphi_{a, i}$ is actual angle of the $i$ th joint, and $K_{i}$ is stiffness of the $i$ th joint.

Darainy et al. (2004) through their measurements have shown that joint stiffness has a dynamic property and changes in the course of the movement. However, for the sake of simplicity, in this study the joint stiffness is assumed to be constant during movement, which implicitly means that the (described by (3)) and a responsibility estimator (described by (4)). Each auxiliary module cooperates autonomously in generating the control signal

co-activation command is also supposed to be constant. This assumption is justifiable, because, there are studies in which the joint stiffness is assumed to be constant during the movement and they still provide reasonable results (Greene and McMahon 1979; Mizrahi and Susak 1982; Ozguven and Berme 1988; Kim et al. 1994; Farley et al., 1998 Spagele et al. 1999).

\subsection{Controllers and modules}

The CNS can learn and control a new movement in different contexts using its previous experiments and through repeating new tasks. To describe this feature in the proposed model, MODEM consists of several subordinate experts 
which collaborate in controlling the task according to the weights assigned to them by a "gate selector" in a higher level (Fig. 1A). In this architecture, the knowledge of controlling a task is divided between the experts with regards to the nature of the task. Each expert is specialized in controlling a part of the task or the task under special conditions and constraints. On the other hand, the "gate selector" block assigns the control of the task to a subordinate expert according to the contextual information which is feed-back by sensory information signals or by top-down information from higher-motor-related areas. Therefore, enough knowledge or well-defined criteria must be embedded in the "gate selector" block. In fact, the "gate selector" codes the knowledge of the control of different dynamics of the system into the experts. In other words, the "gate selector" confers the control of the motor system to the appropriate expert under current conditions (or switched between experts). The details for a case study will be explained in Sect.3.3.

Since there are delays in the pathway of the sensory information feedback in the biological system, a delay block is also considered to model the whole structure (see Fig. 1A). 100-ms delay was set for all of the joints (Kandel et al. 2000).

The structure of the experts is illustrated in more detail in Fig. 2. The architecture of each expert is modular. There are several auxiliary modules in each expert. This is one of the main differences between MODEM and the common structure of MEX. In MODEM, each expert consists of a main controller and a main predictor as well as the auxiliary modules. In each expert, the main controller generates the main central command and the auxiliary modules correct the central command when it fails to produce an appropriate command, especially when there is an external disturbance. The number of auxiliary modules is equal to the number of degrees of freedom (DOF) of the motor system; i.e., in each expert, each auxiliary module is responsible for $1 \mathrm{DOF}$. The correction signal of an auxiliary module depends on the responsibility of that module which in turn is a function of the similarity of the main predictor and corresponding predictor of that auxiliary module.

The main controller of each expert generates the original central command to control the task. To compensate deviations from desired movements due to unpredicted disturbances or other reasons, each auxiliary module will contribute independently in correcting the main central command according to its corresponding degree of responsibility. The responsibility of each module determines the amount of cooperation of that module. In other words, each auxiliary module contributes autonomously in generation of the central command. Since the auxiliary modules are autonomous, they are considered as agents.

What we called controller (main controller of each expert and the controller of each agent) is in fact an inverse model of the body-skeletal dynamics together with the JCC (named "the model of motor system" in Fig. 1A). The input of each controller block is the desired movement, i.e., the joint angle and the angular velocity and acceleration of the joints.

The central command generated by MODEM is adjusted by the feedback command $\left(R_{\mathrm{fb}}\right)$. The main controller of each expert generates the main central command only using the feed-forward information, i.e., the desired movement. The output signals of the auxiliary modules are generated using the information of the desired and actual movements, and the efference copy.

Linear networks are used to implement the controllers. A linear network is a Multi-Layer Perceptron (MLP) network, i.e., a kind of artificial neural network with linear activation function and without any hidden layers. While, the controllers are realized using linear networks, we will see later that the auxiliary modules adjust the central command through nonlinear relations.

The inputs of the main predictors are sensory information (actual movement) and the delayed efference copy generated by that expert (reciprocal command). The inputs of the predictor of each auxiliary module are delayed desired movement and the reciprocal command of its corresponding expert (see Fig. 2). The outputs of the main predictor and the predictors of the auxiliary modules of the $j$ th expert are all joint torques and are determined by Eqs. 2 and 3, respectively.

$$
\begin{aligned}
\hat{\tau}_{\mathrm{a}, j}^{k} & =K_{k}\left(\varphi_{\mathrm{a}, k}-R_{j}^{k}\right) \\
\hat{\tau}_{i, j}^{* k} & =K_{k}\left(\varphi_{k}^{*}-R_{j}^{k}\right)
\end{aligned}
$$

where $\hat{\tau}_{\mathrm{a}, j}^{k}$ and $\hat{\tau}_{i, j}^{* k}$ are the $k$ th element of the estimated joint torques generated by the main predictor and the predictor of the $i$ th module in the $j$ th expert, respectively. $k$ is between 1 and $m ; m$ is the total number of the joints. $R_{j}^{k}$ is the $k$ th element of the reciprocal command vector generated by the $j$ th expert. $\varphi_{\mathrm{a}, k}$ and $\varphi_{k}^{*}$ are the actual and desired angle of the $k$ th joint, respectively. $K_{k}$ is the stiffness of the $k$ th joint.

The responsibility of each module is calculated by Eq. 4 .

$\lambda_{i j}=0.5+0.5 \tanh \left(\frac{\left|\hat{\tau}_{\mathrm{a}, j}^{i}-\hat{\tau}_{i, j}^{* i}\right|}{W_{i j}}-T_{i j}\right)$

where $\lambda_{i j}$ is the responsibility factor of the $i$ th module of the $j$ th expert. $T_{i j}$ (threshold) and $W_{i j}$ (weight) are tuning factors to adjust the quality of cooperation of each joint. The responsibility of an agent can be very small when the similarity between the output of the main predictor and the predictor of that agent is high. In other words, the agents have more influence in generating the commands when the main controller cannot control the task properly.

The total central command is the weighted summation of the output of the main controller plus the outputs of controllers of agents each weighted by the responsibility signal $\lambda_{i j}$ and a scaling factor: 


$$
\begin{aligned}
R= & \sum_{j=1}^{n} G_{j} \\
& \times\left(R_{0 j}+\sum_{i=1}^{m} R_{i j} \lambda_{i j} S_{i j}\left(\varphi_{i}^{*}\left(t-t_{\mathrm{d}}\right)-\varphi_{\mathrm{a}, i}^{\left(t-t_{\mathrm{d}}\right)}\right)\right)
\end{aligned}
$$

where $R_{0 j}$ is the main central command generated by main controller of $j$ th expert. $R_{i j}$ and $\lambda_{i j}$ are the central command and responsibility factor corresponding to $i$ th modular controller of $j$ th expert, respectively. $G_{j}$ is a weight assigned to the $j$ th expert by the "gate selector" to show the contributions of that expert. $\varphi_{i}^{*}$ and $\varphi_{\mathrm{a}, i}$ are desired and actual movements of the $i$ th joint, respectively and, $S_{i j}$ and $t$ are the scaling factor and time variable, respectively.

\subsection{Learning method}

In MODEM, it is assumed that the desired movement is already known to the motor control system (already developed in the motor planning level) and it is considered as a prior knowledge which is embedded in the system.

On the other hand, the appropriate number of experts is chosen based on the changes in the dynamics of the system in interaction with its environment. Therefore, we divide the data into different classes, each class corresponding to one part of the task which is defined according to the interaction of the motor system with its environment. Each class or part is called a subtask. Therefore, for each expert the data belonging to the corresponding subtask should be used to train its controllers.

Inspired by the idea of the movement primitives (Schaal 2002; Schaal et al. 2003), we assumed that the experts have learnt the control of the corresponding subtasks separately, and later these experts were coordinated by a higher level critic to control a more complex task under new conditions. Therefore, we used a sequential learning method to train different components of the experts. As the first step, the experts are trained in the absence of the agents. In fact, the main controllers play the most important role in generating the control signal. We do not change the parameters of the main controllers after this part of learning. The controllers are trained using a supervised learning algorithm with error back-propagation method. In this study, we used a set of recorded data (see Sect.3.3) to represent the desired movement. The desired outputs of the controllers have been calculated from these data. This technique is called teacher forcing technique (Williams and Zipser 1989; Doya and Yoshizawa 1989). This method is an efficient technique for autonomous dynamic systems.

To train the controllers, we need the desired central command $\left(R^{i^{*}}(t)\right)$. It is assumed that desired joint torque at $i$ th joint $\left(\tau_{i}^{*}\right)$ corresponds to the desired central command $\left(R^{i^{*}}\right)$.
Knowing the desired movement $\left(\varphi_{i}^{*}\right)$ and stiffness of $i$ th joint, the reciprocal command of that joint can be calculated by (6).

$R^{i^{*}}(t)=\varphi_{i}^{*}(t)-\frac{\tau_{i}^{*}(t)}{K_{i}}$

$R^{i^{*}}(t)$ is used to train the controllers. The desired joint torque is calculated from dynamical equations of the body-skeletal dynamics for the desired movement (see Sect. 3.2).

The data used to train the main controller of an expert are the desired movement (consisting of desired joint angles and their corresponding angular velocities and accelerations) and its corresponding reciprocal command (see Fig. 2). In this case, the output is the central command vector.

In the next step, after the training of the main controllers is completed, the auxiliary modules are to be trained one by one. Each time a constant disturbance is applied in one of the joints and the corresponding auxiliary module in each expert is trained under this new condition. In fact, the corresponding auxiliary module in each expert learns to generate a correction command, so that the movement is performed correctly under the new condition. Finally, after all auxiliary modules have been trained separately, MODEM is ready to control the task with or without external disturbance.

\section{Validation of the model through simulation}

We investigate the performance of the proposed architecture through simulation studies. For this purpose, we will study the ability of MODEM to control a given movement (task) robustly in the presence of external disturbances applied on the motor system, noise in sensory information, and changes in the environment. We also compare performance of MODEM in the absence of its agents with that of the MOSAIC model.

\subsection{Chosen task}

The chosen task is STS. In most of previous studies in the field of motor control modeling, researchers have considered reaching movements as a case study (Bhushan and Shadmehr 1999; Haruno et al. 2001; Haruno et al. 2003). The proposed structure (MODEM) is well-suited for controlling complex tasks that consist of different subtasks. Therefore, we decided to study a task including distinguishable subtasks. There are different studies demonstrating the existence of different subtasks for the STS transfer (for example Emadi Andani et al. 2007 and 2009); whereas, reaching movement is a very simple task in comparison to STS and most probably (according to the mentioned criteria in the previous section) it consists of only one single subtask. Consequently, we considered STS as our case study. It should be noticed that dynamic stability of the whole motor system is an important issue during STS 
task performance; this issue does not come into question for a reaching task. This makes the control of STS more challenging than the reaching task. As we will see later, dynamical stability considerations (relative position of the body center of pressure) plays an important role in the adjustment of model parameters $\left(S_{i j}, T_{i j}\right.$, and $\left.W_{i j}\right)$.

We distinguish two main phases of STS based on the interaction of the body (motor system) with its environment: (I) before leaving the seat, when the body is in contact with the seat; (II) after leaving the seat, when the body has no contact with the seat and is extending upward toward standing posture. Therefore, the moment when the body leaves the seat, i.e., the seat-off moment, is a crucial moment at which the motor system switches between these two phases (transferring from phases I to II). Later, we will see that the higher level "gate selector" learns a simple criterion to separate these two phases.

\subsection{Model of the system and recorded data}

The human motor system consists of the muscle dynamics and the body-skeletal dynamics. At each joint, two groups of muscles (agonists and antagonists) generate the necessary torques in cooperation. To avoid the complexities resulting from choosing the suitable muscle model as well as the problem of intrinsic redundancy of the number of muscles controlling each joint, we considered only the body-skeletal dynamics when modeling the motor system. All the mentioned complexities could result in questions which were not our main concerns in this article. However, the JCC block represents the overall function of the agonist and antagonist muscles in generating necessary joint torques.

Following our previous study (Emadi Andani et al. 2007), a four-link 3DOF model including feet, shank, thigh, and upper body in the sagittal plane was developed to describe body-skeletal dynamics. The feet were assumed to remain fixed on the floor (Fig. 3). Each joint has 1DOF. Before leaving the seat, the contact between the body and seat was modeled as a soft point-contact. It was assumed that the two reaction forces in the horizontal and vertical directions exerted by the soft seat are acting only on the hip joint (Fig. 3). These forces were generated based on two couples of nonlinear spring-damper models (Davidson and Wolpert 2004) described by (7) and (8).

$F_{y \mathrm{ch}}=\left\{\begin{array}{cc}\left(K_{y}-B_{y} \dot{y}\right)\left(e^{-a_{y}\left(y-y_{0}\right)}-1\right) & F_{y \mathrm{ch}}>0 \\ 0 & F_{y \mathrm{ch}} \leqslant 0\end{array}\right.$
$F_{x \mathrm{ch}}=\left\{\begin{array}{cc}K_{x}\left(e^{-a_{x}\left(x-x_{0}\right)}-1\right)-B_{x} \frac{\dot{x}}{y-y_{0}+0.1} & F_{y \mathrm{ch}}>0 \\ 0 & F_{y \mathrm{ch}} \leqslant 0\end{array}\right.$

where $F_{x \mathrm{ch}}$ and $F_{y \mathrm{ch}}$ are the chair reaction forces in horizontal and vertical directions, respectively. $K_{x}, K_{y}, B_{x}, B_{y}, a_{x}$,

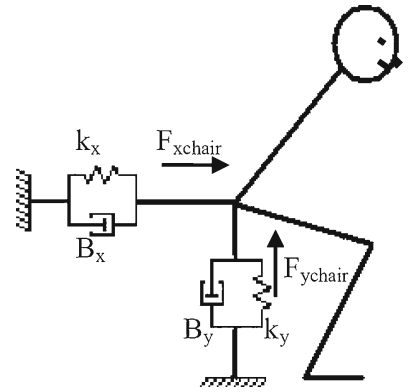

Fig. 3 Four link rigid model of the body. Reaction forces exerted by the seat are generated by two pairs of nonlinear spring-damper (Emadi Andani et al. 2007)

and $a_{y}$ are constant parameters. The dot sign show the first derivative and, $x_{0}$ and $y_{0}$ are the rest position of the spring models in horizontal and vertical positions, respectively.

Using Newton-Euler equations three nonlinear differential equations were obtained to describe the dynamics of the body-skeletal model during STS transfer (Hemami 1982; Craig 1986). The necessary anthropometric parameters of the body-skeletal dynamics including segmental mass, the distance of the center of mass of segments from distal joints, and the inertial constants of each segment were estimated by a bilinear regression formula using the body height and weight (Zatsiorsky and Seluyanov 1983).

The data to train and test the proposed structure were collected at the Gait and Posture Laboratory of University of Waterloo (Moraes et al. 2002). Data included the trajectories of joint angles of one side of the body of one subject in the sagittal plane. The subject has repeated the task 20 times. Sampling frequency of data was $60 \mathrm{~Hz}$.

All recorded trajectories were aligned based on the seatoff moment. Then for each repetition of the task, the data between $1.5 \mathrm{~s}$ before seat-off and $1.5 \mathrm{~s}$ after seat-off were chosen. Ten sets of data were used to train the model (named training data) and the rest of the data were used to test the model (named test data). The joint torques corresponding to desired movements are calculated using the Newton-Euler equations; Eqs. 7 and 8 are the external forces applied at the hip joint before seat-off. Anthropometric parameters of the subject were used in the dynamical equations of the motor system. Subject's weight and height were $57.4 \mathrm{~kg}$ and $1.71 \mathrm{~m}$, respectively. Assuming constant stiffness for each joint, the desired reciprocal commands were calculated and used to train the controllers using (6).

\subsection{Simulation of the learning procedure}

The STS task is made of two main phases; before and after leaving the seat. Our previous simulation results (Emadi Andani et al. 2007) confirmed this partitioning. In that study, we proposed an automatic learning method for the MOSAIC structure, which extracts the constitutive subtasks of a given 
movement, and we applied the method to the STS task. The results confirmed that there are two main subtasks in the STS. One of the important results obtained in that study was that the switching moment between the two subtasks is the seatoff moment. Therefore, two main controllers, one per expert, are needed to control this task: one before seat-off and the other after seat-off moment. Therefore, to control the STS task, two experts $(n=2)$, each with one main controller, one main predictor, and three auxiliary modules corresponding to the three joints $(m=3)$ is needed.

The higher level "gate selector" selects the appropriate expert by sensing the seat-off moment. This information is extracted from the sensory data fedback by the proprioceptors. According to this criterion, the task must be controlled by the first expert before seat-off moment, and the second expert has to control the task after this crucial moment. We considered hard switching between the experts, i.e., the value of $G_{j}$ is either 0 or 1 . Therefore, at any time only one expert performs the control of the task. In this study, $G_{j}$ is one (zero) and zero (one) for the first (second) expert before (after) leaving the seat. The value of $G_{j}$ is determined through the delayed sensory feedback about the hip contact with the seat.

The data partitioned into two classes with reference to the seat-off moment and each class of data was applied to train the corresponding main controller of the experts, separately. In the training data of the two experts, an overlap about the switching moment was created. To train the first expert, in addition to the pre-seat-off data, the data of movement up to $150 \mathrm{~ms}$ after seat-off were also taken into account. The first expert is responsible for controlling the movement before seat-off. In fact, the first expert is forced to learn the beginning of the sub-task 2 to compensate for the uncertainty resulting from the sensory delay. In this way, the controller of the first sub-task will not be any more sensitive to the exact detection of the seat-off moment, or in other words to the exact moment of the switching between two control modules. In this case, the continuity of the central command will also be hold.

The central commands generated by the main controller of the experts are shown in Fig. 4. As Fig. 4 indicates, central commands generated by the two experts are identical for ankle and knee joints between seat-off and the perception of the seat-off by the CNS. Perception of the seat-off occurs with a delay of $100 \mathrm{~ms}$ relative to the actual seat-off (delay in the sensory feedback). For the hip joint, the range of identical functioning of the two experts is vaster, i.e., to control this joint, principally, one expert is sufficient. In other words, principles governing the movement of the upper trunk before and after seat-off are almost the same.

In Fig. 5, one can see the torques generated as a result of the central commands of the main controllers. The results indicate that the joint torques and output of the feedback controllers are continuous during the movement.
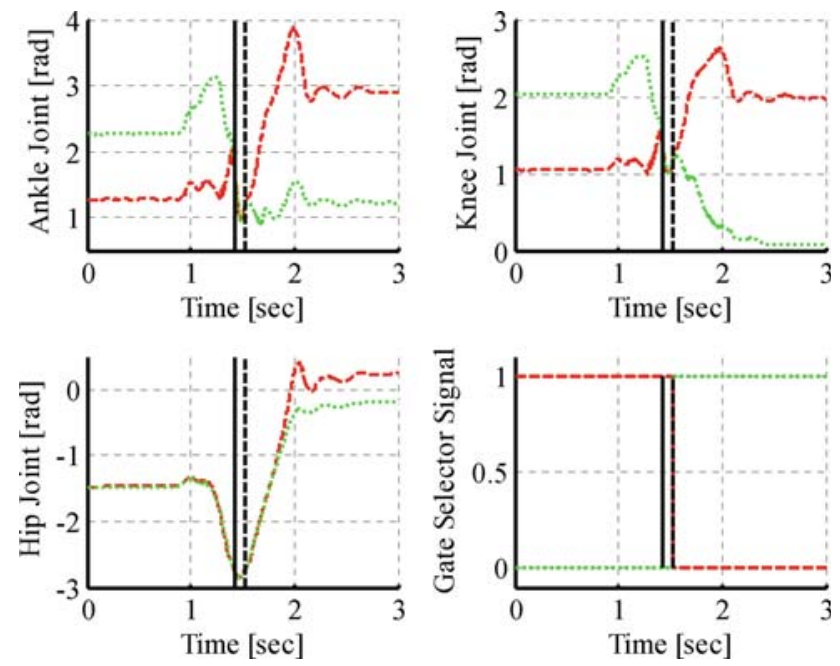

Fig. 4 Central commands generated by the main controller of expert \#1 (red dashed line) and expert \#2 (green dotted line) for each joint are shown separately. The seat-off moment and its perception, which is occurred with a delay of $100 \mathrm{~ms}$, are shown by solid and dashed vertical black lines, respectively. Seat-off is occurred when vertical seat reaction force is equal to zero. Output of the gate selector for each expert is shown on bottom-right figure
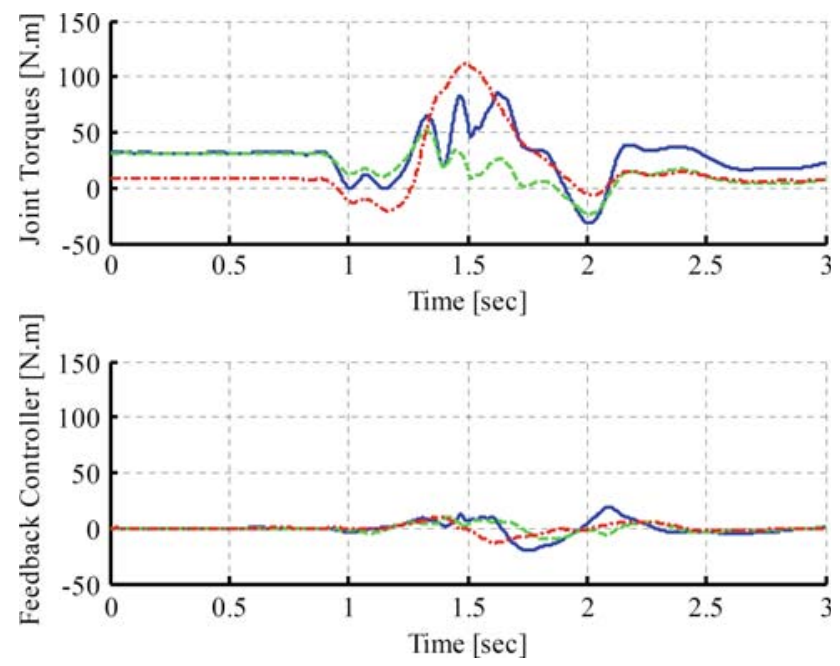

Fig. 5 Torques generated by MODEM controller and corresponding feedback controller (condition 1-2 of the Table 1). The torques corresponding to ankle, knee, and hip joints are shown by solid blue line, dashed green line and dashed-dot red line, respectively

After training the main controller of the experts, a $20 \mathrm{Nm}$ constant external disturbance was exerted at each joint separately, and the training of the controllers of the corresponding agents in each expert was completed.

\subsection{Adjusting parameters of the model}

The parameters of the feedback controller were determined through a trial and error procedure after training the MODEM structure in order to decrease the activity of the feedback 
controller (see (10)) and at the same time increase dynamical stability for the training data.

The feedback controller block in Fig. 1, which in fact consists of three separate feedback controllers, one for each joint (hip, knee, and ankle) is described by Eq. 9 .

$$
R_{\mathrm{fb}}=K\left(\varphi^{*}-\hat{\varphi}_{\mathrm{a}}\right)+D\left(\dot{\varphi}^{*}-\hat{\dot{\varphi}}_{\mathrm{a}}\right)
$$

where $K(=\operatorname{diag}[1.7,1.7,1.7]), D(=\operatorname{diag}[0.8,0.8,0.8])$, and $R_{\mathrm{fb}}$ are diagonal $3 \times 3$ matrices. $\varphi^{*}$ (desired joint angle), $\dot{\varphi}^{*}$ (desired angular velocity of joint), $\hat{\varphi}_{\mathrm{a}}$ (predicted joint angle) and $\hat{\dot{\varphi}}_{\mathrm{a}}$ (predicted angular velocity of joints) are $3 \times 1$ vectors.

After training the controllers and choosing the parameters of the feedback controller, we adjusted $S_{i j}, W_{i j}$, and $T_{i j}$ to provide the dynamical stability of the movement for training data in the presence of a constant external disturbance. Dynamical stability means to keep the center of pressure during movement under the base of support. It was assumed that these parameters were constant during task performance. However, we considered identical scaling factor $\left(S_{i j}=100\right)$, normalizing factor $\left(W_{i j}=4\right)$, and threshold $\left(T_{i j}=2\right)$ for all modules.

\subsection{Simulation studies}

Performance of the proposed model was investigated under several conditions. Three control models were considered: (1) with only a feedback controller to control the task, (2) with a feedback controller and the MODEM with two experts and no auxiliary modules; in this case, the structure is equivalent to the MEX model. Finally, (3) with a feedback controller and the MODEM. We studied the performance of the three mentioned structures when controlling the STS transfer.

It was observed that the simple feedback controller could not control the task and the system was unstable. This observation is not surprising, because there is no proof for global asymptotic stability of a linear PID controller when controlling a robot manipulator (Loria et al. 2000). Therefore, the performance of this model will not be included in our discussions.

Two criterions to compare the performance of the two other structures, i.e., MODEM and MEX, are defined: (I) the level of the Activity of the Feedback Controller $(A F C)$ (defined by (10)), (II) the tracking error (defined by (11)).

$$
\begin{gathered}
\mathrm{AFC} \triangleq \sqrt{\frac{\int_{0}^{t_{\mathrm{f}}}\left(\tau_{P D}(t)\right)^{2} \mathrm{~d} t}{t_{\mathrm{f}}}} \\
\text { Tracking error } \triangleq \sqrt{\frac{\int_{0}^{t_{\mathrm{f}}}\left(\varphi_{\mathrm{a}}(t)-\varphi^{*}(t)\right)^{2} \mathrm{~d} t}{t_{\mathrm{f}}}}
\end{gathered}
$$

The $A F C$ is an indicator for the ability of the controllers in controlling the task, especially in the presence of disturbance. The tracking error is an indicator for the quality of the generated movement.

\subsubsection{Simulation experiment I: disturbance rejection}

In the first group of simulation studies, we investigated the performance of MODEM without any external disturbance, and in the presence of slow and fast-dynamic external disturbances (sinusoidal signals) applied at the shoulder. The frequencies of the external sinusoidal disturbance were considered $1 / \pi \mathrm{Hz}$ (as the slow dynamic) and $3 / \pi \mathrm{Hz}$ (as the fast dynamic).

Six conditions are tested, and the results are summarized in Table 1. The six conditions (1-1 to 1-6) correspond to two structures for the motor controller (the MODEM and the MEX) and three cases of no external disturbance, and slow and fast external disturbances at the shoulder.

Figure 6 compares the effect of the feedback controller in generating the compensatory torque under conditions 1-1 and 1-2 for a test data. As it can be seen the activity of the feedback controller after stabilizing the movement when the MODEM architecture is used is significantly smaller than when the MEX is used (see Fig. 6, for time $>2.5 \mathrm{~s}$ ).

Comparing the results of conditions 1-1 and 1-2 in Table 1, one concludes that the control of the task with no external disturbance is performed more accurately by the MODEM architecture. Looking into the results of conditions 1-3 and 1-4 in Table 1, one concludes that the control of the task in the presence of external disturbance with slow dynamic at the shoulder is performed more accurately by the MODEM architecture. Also, the results of condition 1-5 and 1-6 in the Table 1 show that the control of the task in presence of external disturbances with fast dynamic at the shoulder is performed more accurately by the MODEM architecture. In addition, the MEX structure cannot control the task in the presence of large slow/fast-dynamic external disturbances, while the MODEM can accomplish the task. It was also observed that the amplitude of the external disturbance for conditions 1-3 and 1-5, when MEX is the controller, was crucial. The system was not stable if the amplitude was more than $10 \mathrm{Nm}$ (for slow dynamics) and $3 \mathrm{Nm}$ (for fast dynamics). On the contrary, using the MODEM model, the system was stable in the presence of disturbances with higher amplitudes. In this case, the amplitude of the disturbance could be increased up to $40 \mathrm{Nm}(30 \mathrm{Nm})$ for the external sinusoidal disturbance with slow (fast) dynamics. In summary, it was observed that the MODEM structure results in smaller tracking errors and $A F C$ factor when compared to the MEX (see Table 1). In addition, the MODEM structure was more stable and robust in the presence of larger external disturbances in comparison to the MEX. 
Table 1 The level of the AFC and the tracking error of the motor controller when MODEM and MEX structure control STS transfer with and without external disturbance (applied at the shoulder)

\begin{tabular}{|c|c|c|c|c|c|c|c|c|}
\hline \multirow[t]{2}{*}{ Shoulder disturbance } & \multirow[t]{2}{*}{ Controller } & \multirow{2}{*}{$\begin{array}{l}\text { Index of the } \\
\text { condition }\end{array}$} & \multicolumn{3}{|c|}{ AFC $(\mathrm{Nm})$} & \multicolumn{3}{|c|}{ Tracking error $\left({ }^{\circ}\right)$} \\
\hline & & & Ankle & Knee & Hip & Ankle & Knee & Hip \\
\hline \multirow[t]{2}{*}{ No external load } & MEX & $1-1$ & 7.6 & 5.8 & 3.9 & 1.7 & 0.9 & 0.8 \\
\hline & MODEM & $1-2$ & 6.6 & 3.7 & 3.9 & 0.4 & 0.2 & 0.4 \\
\hline \multirow[t]{2}{*}{ Slow-dynamic external load amplitude $=10 \mathrm{~N} \mathrm{~m}, f=1 / \pi \mathrm{Hz}$} & MEX & $1-3$ & 6.4 & 9.1 & 18.9 & 1.0 & 2.3 & 3.7 \\
\hline & MODEM & $1-4$ & 4.6 & 3.3 & 3.7 & 0.3 & 0.2 & 0.5 \\
\hline \multirow[t]{2}{*}{ Fast-dynamic external load amplitude $=3 \mathrm{Nm}, f=3 / \pi \mathrm{Hz}$} & MEX & $1-5$ & 6.5 & 5.0 & 4.3 & 1.5 & 0.7 & 0.6 \\
\hline & MODEM & $1-6$ & 6.6 & 3.7 & 3.7 & 0.4 & 0.2 & 0.4 \\
\hline
\end{tabular}

External disturbances are sinusoidal signals with $f=1 / \pi$ (slow) and $3 / \pi$ (fast) Hz. Attributes slow and fast were used to distinguish rate of changes of the disturbance signals in time domain

Fig. 6 The effects of the feedback controller in generating the compensative torques are illustrated in $\mathbf{A}$ : condition 1-1 and $\mathbf{B}$ : condition 1-2 (ankle (solid blue line), knee (dashed green line) and hip (dotted dashed red line) joints)
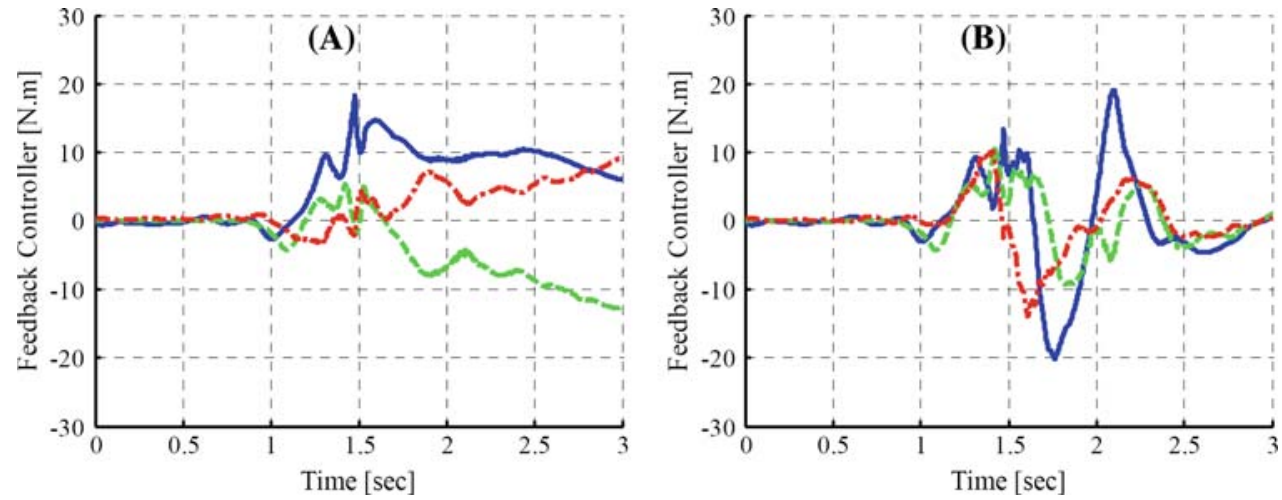

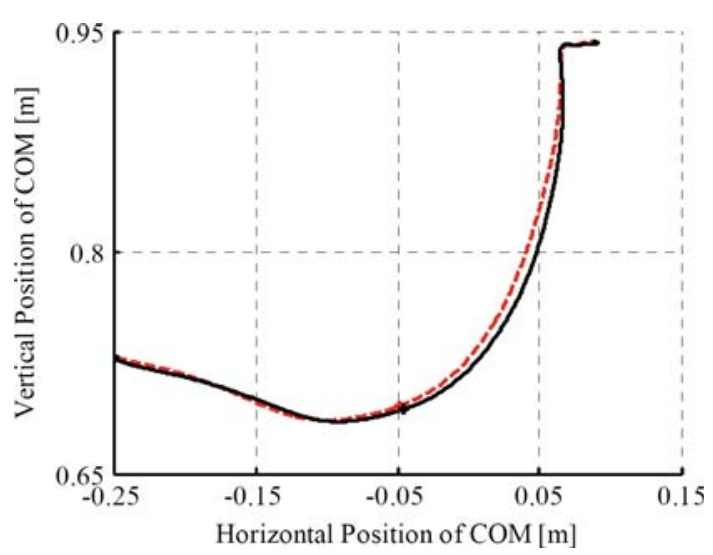

Fig. 7 The desired (dashed red line) and actual (solid black line) position of the COM. Seat-off moment is specified by asterisk. The results are corresponding to condition 1-4 of the Table 1

Figure 7 shows the trajectory of the COM corresponding to desired (dashed red line) and actual (solid black line) movement in the sagittal plane for condition 1-4 of Table 1.

\subsubsection{Simulation experiment II: environmental change}

The next experiment considers the control of the task for the test data in a different environment. This study simulates the case when one learns to stand up from a given seat and then the characteristics of the seat are changed. The elasticity factor of the chair in vertical direction ( $K_{y}$ in (7) and Fig. 3) is an important parameter because it carries a main part of the body weight before the seat-off. In this case, we changed $K_{y}$ and tested the model for the MODEM and the MEX architectures: $K_{y}$ was increased by $100 \%$ and decreased by $20 \%$. The results are summarized in Table 2. All controllers are trained for the same value of the vertical elastic parameter of that chair.

Table 2 shows that in the presence of uncertainties in the environment, the MODEM architecture results in more accurate tracking of the desired movement than the MEX. It must be mentioned that the movement was not stable if the elasticity of the seat was increased more than $100 \%$ or decreased less than 20\% for the MEX, but the MODEM could control the task for a wider range of values. In addition, comparing the tracking errors depicted in Table 1 (conditions 1-1 and 1-2, when the environment has no uncertainty) and Table 2 (when the environment is different from what has been learnt before), it is concluded that MODEM tracks the desired movement for different unpredicted variations in the vertical elasticity of the chair with almost the same tracking error as in the case of the original environment.

\subsubsection{Simulation experiment III: robustness against noise}

The next group of simulations considers the control of the task in the presence of noise in the sensory feedback when the test data was used. In this case, we considered $10 \%$ of additional signal-dependent normal noise in the sensory information from joint angles and angular velocities. We tested the 
Table 2 The level of the AFC and the tracking error of the motor controller when MODEM and MEX structure control STS transfer with different chairs for two modes of controller

\begin{tabular}{|c|c|c|c|c|c|c|c|c|}
\hline \multirow[t]{2}{*}{$K_{y}$} & \multirow[t]{2}{*}{ Controller } & \multirow[t]{2}{*}{ Index of the condition } & \multicolumn{3}{|c|}{$\mathrm{AFC}(\mathrm{Nm})$} & \multicolumn{3}{|c|}{ Tracking error $\left(^{\circ}\right)$} \\
\hline & & & Ankle & Knee & Hip & Ankle & Knee & Hip \\
\hline$+100 \%$ & MEX & $2-1$ & 9.2 & 9.2 & 5.1 & 1.9 & 1.6 & 0.8 \\
\hline$+100 \%$ & MODEM & $2-2$ & 12.6 & 6.9 & 12.4 & 0.5 & 0.3 & 0.4 \\
\hline$-20 \%$ & MEX & $2-3$ & 30.2 & 26.6 & 16.3 & 6.9 & 4.5 & 2.3 \\
\hline$-20 \%$ & MODEM & $2-4$ & 16.5 & 7.6 & 6.6 & 0.9 & 0.5 & 0.4 \\
\hline
\end{tabular}

model 50 times for each of the MODEM and MEX structures and the results including mean values and standard deviations of the AFC and tracking errors are depicted in Table 3. The desired (red dashed line) and the mean trajectories of the 50 movements (black solid lines) are illustrated in Fig. 8A and $\mathrm{b}$ when the MODEM and the MEX structures were tested, respectively. The green area shows the variability of the movement for the 50 trials in the presence of noise (see Fig. 8). It is also seen that the green area in Fig. 8A is narrower than the corresponding area in Fig. $8 \mathrm{~B}$. The results of Table 3 also show that the MODEM architecture reduced significantly the tracking error and its standard deviation.

In general, the results show that the MODEM architecture is more robust against noise in sensory information than the MEX.

\subsubsection{Simulation experiment IV: robustness against uncertainty in the forward model of motor system}

Unquestionably the quality of the forward model of motor system (see Fig. 1) which is a component of the inner loop of a Smith predictor influences the function of the feedback controller. It means that inaccuracy of the forward model results in increasing $A F C$ as well as increasing the tracking error and finally decreasing the dynamical stability. For this reason, the performance of the proposed structure was studied in the presence of uncertainty in the forward model of motor system.

To show the performance of the MODEM with inaccuracy in the forward model, we add normal noise to the stiffness $(K)$ of each joint in the forward model of the motor system. This noise will model up to $20 \%$ variation in the stiffness of the joints. The results are depicted in the Table 4. Comparing indices of conditions 1-1 and 1-2 of the Table 1 and 4-1 and 4-2 of the Table 4, one concludes that the $A F C$ is increased in the presence of the noise but the tracking errors do not show any significant changes.

On the other hand, adding the same inaccuracy in the forward model of the motor system when the MEX is as the combined controller, one observes that the $A F C$ of the knee joint is increased from $5.8 \mathrm{Nm}$ (Table 1) to $7.5 \mathrm{Nm}$ (Table 4), while the tracking errors are $0.9^{\circ}$ for accurate forward model and $0.9 \pm 0.3\left(^{\circ}\right)$ for inaccurate forward models. For a similar situation, when the MODEM is as the combined controller, the $A F C$ of the knee joint is increased from $3.7 \mathrm{Nm}$ (Table 1) to $6.8 \mathrm{Nm}$ (Table 4$)$, while the tracking errors are about $0.2\left(^{\circ}\right)$ for both the conditions. An important issue is that the standard deviations of tracking error are less when the MODEM is used, i.e., the agents decreased the variability of the movement significantly and thus result in more robustness.

\section{Discussion}

In the MODEM structure, different important features of the human motor control are brought together to develop a model which behaves more in conformity with the recorded observations about human behaviors during a ballistic task performance than previously developed models. That is why two new features were added to the previously suggested structures. In fact, the ideas of a hierarchical control structure and multiple internal models as well as that of EPH are not new in this field (Feldman and Latash 2005). Whereas, in the MODEM structure, the idea of the reciprocal command is integrated into the structure of a hierarchical MEX, in which each expert is a paired forward-inverse model. Previous models, like MOSAIC, were based on internal models and did not show and explain the role of the reciprocal command in the movement control. Joint torques generated based on the reciprocal command (see (1)) results in an internal and innate feedback at the motor system which provides more stability for the movement (Feldman and Latash 2005). The second new feature of MODEM is disturbance rejection by agents with generalization capability.

In this section two aspects of the proposed model will be discussed: (1) the MODEM architecture provides a consistent formulation of how modular internal models can cooperate to perform a sequential movement and increase the robustness against external disturbances, changing environment and, additional signal-dependent noise in the sensory information; and then (2) the behavioral and the neurophysiological supports of this structure.

First, we would like to discuss about the efficiency of the MODEM structure in controlling any given task. The local feedback loop in Fig. 1, consisting of the forward model of the motor system and the feedback controller, is in fact the inner loop of a Smith predictor structure. The 


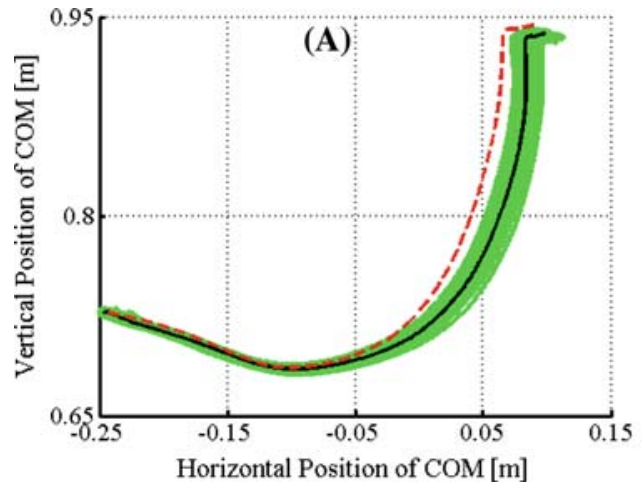

Fig. 8 A The position of the COM of a performed movement which was controlled using the MEX. B The position of the COM of a performed movement which was controlled using the MODEM.

The COM of desired movement is shown by dashed red line, the mean

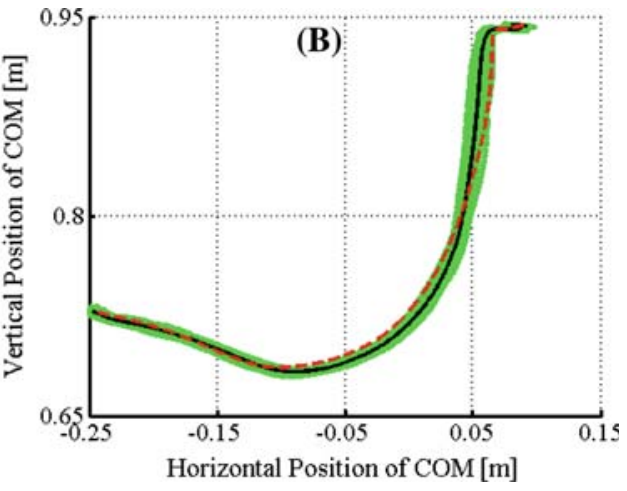

of several performed movement under noise in sensory information is specified by black solid line, and the range of movement variability performed in 50 trials is shown by shadow green area

Table 3 Mean value and standard deviation of AFC and tracking error of 50 different trials of the STS transfer in the presence of $10 \%$ additional signal-dependent noise in sensory information for two modes of controller

\begin{tabular}{|c|c|c|c|c|c|c|c|}
\hline \multirow[t]{2}{*}{ Controller } & \multirow[t]{2}{*}{ Index of the condition } & \multicolumn{3}{|l|}{$\mathrm{AFC}(\mathrm{Nm})$} & \multicolumn{3}{|c|}{ Tracking error $\left(^{\circ}\right)$} \\
\hline & & Ankle & Knee & Hip & Ankle & Knee & Hip \\
\hline MEX & $3-1$ & $14.0 \pm 2.08$ & $18.9 \pm 1.82$ & $12.9 \pm 1.82$ & $2.12 \pm 0.54$ & $2.14 \pm 0.51$ & $1.67 \pm 0.45$ \\
\hline MODEM & $3-2$ & $14.9 \pm 1.93$ & $16.3 \pm 1.22$ & $12.7 \pm 1.30$ & $0.47 \pm 0.10$ & $0.31 \pm 0.05$ & $0.36 \pm 0.04$ \\
\hline
\end{tabular}

Table 4 Mean value and standard deviation of $A F C$ and tracking error of 10 different trials of the STS transfer in the presence of $20 \%$ additional signal dependent noise in the stiffness $(K)$ of the joints for two modes of controller

\begin{tabular}{lllllllll}
\hline Controller & Index of the condition & AFC $(\mathrm{Nm})$ & & & \multicolumn{3}{l}{ Tracking error $\left(^{\circ}\right)$} \\
\cline { 3 - 4 } & & Ankle & Knee & Hip & & Ankle & Knee & Hip \\
\hline MEX & $4-1$ & $10.4 \pm 1.8$ & $7.5 \pm 1.5$ & $6.1 \pm 1.4$ & & $1.9 \pm 0.4$ & $0.9 \pm 0.3$ & $1.0 \pm 0.3$ \\
\hline MODEM & $4-2$ & $10.9 \pm 1.4$ & $6.8 \pm 0.7$ & $8.9 \pm 1.3$ & $0.4 \pm 0.07$ & $0.2 \pm 0.03$ & $0.4 \pm 0.06$ \\
\hline
\end{tabular}

agents (auxiliary modules) of each expert represent nonlinear disturbance observers; in fact, they generalize the role of the external feedback loop of a Smith predictor. These two types of controllers, i.e., the feedback controller (Fig. 1) and the other controllers in the auxiliary modules (Fig. 2), contribute differently in controlling the task, because they have access to different types of the information: the feedback controller is working based on the predicted joint angle and predicted angular velocity. This loop is needed to compensate the delay of the sensory feedback information. The auxiliary modules are working based on both the delayed sensory feedback information and the desired movement. Since the predicted information might be inaccurate, therefore, another pathway is needed to improve the central commands for controlling the task. The auxiliary modules or agents compensate the error due to inaccuracy of the predicted information.

The advantage of the agents along with the proposed modular structure becomes clearer when comparing the results of
Tables 1, 2, and 3. In Table 1 tracking errors of the MODEM and MEX (MODEM without agents) structures are compared for the test data. The results show that the tracking errors of different joints are smaller when the controller is working together with the agents. Tables 2 and 3 also show that the tracking errors of the MODEM structure are smaller in the presence of uncertainties in the environment and in the sensory feedback compared to the same situations when the MEX structure is used. In summary, the simulation results indicate that the tracking of the movement was performed more robustly when the agents were included in the structure of the controller.

In fact, the agents are modeling the generalization ability of the CNS in rejecting unexpected disturbances. The agents are trained to reject simple constant external disturbances applied at hip, knee, and ankle joints, separately, and then they generalize what they have learnt to more complex external disturbances (like sinusoidal ones) which are applied at another point (like shoulder). 
Table 5 The level of the AFC and tracking error of the motor controller when MOSAIC structure control STS transfer

\begin{tabular}{|c|c|c|c|c|c|c|c|}
\hline \multirow[t]{2}{*}{ Shoulder disturbance } & \multirow[t]{2}{*}{ Index of the condition } & \multicolumn{3}{|c|}{$\mathrm{AFC}(\mathrm{Nm})$} & \multicolumn{3}{|c|}{ Tracking error $\left(^{\circ}\right)$} \\
\hline & & Ankle & Knee & Hip & Ankle & Knee & Hip \\
\hline No external load & $5-1$ & 13.6 & 9.5 & 13.8 & 5.8 & 1.4 & 1.6 \\
\hline Slow-dynamic external load amplitude $=10 \mathrm{Nm}, f=1 / \pi \mathrm{Hz}$ & $5-2$ & 42 & 112 & 6.9 & 13.8 & 21.0 & 1.4 \\
\hline Fast-dynamic external load amplitude $=3 \mathrm{Nm}, f=3 / \pi \mathrm{Hz}$ & $5-3$ & 42.4 & 96.6 & 6.8 & 13.4 & 20.7 & 1.2 \\
\hline
\end{tabular}

To show the advantage of considering the reciprocal command instead of joint torque, we compare the performance of the MEX (MODEM without agents) with the MOSAIC structure with the same number of subtasks.

The results of the simulation for a test data for MOSAIC structure are summarized in Table 5. Comparing the results of the Tables 1 and 5 one concludes that MEX architecture controls the task better than MOSAIC.

Therefore, the existence of the reciprocal command in the structure of MEX results in a control with smaller tracking error. In fact, a structure with the reciprocal command as the central command, integrates the role of the local intrinsic feedback in the motor system due to the JCC structure (see Fig. 1). This feedback provides self control of the movement and in the final analysis generates a more robust motion.

It might be worthy to remember that it is always possible to find another controller structure which can achieve the same or even better performance when compared to MODEM; but the main motivation in developing this structure was its neurophysiological and behavioral supports. There are enough physiological supports in the literature for the first distinctive feature of MODEM, i.e., the role of the reciprocal command (Latash and Gottlieb 1991). Hence, we will try to clarify the second feature (the structure of the agents) more explicitly.

There are different mathematical and behavioral explanations for the function of the agents. Alexandrov et al. (2005) have investigated human balance control during standing through a 3-link, 2-dimensional body-skeletal model in the sagittal plane. Their study included sudden perturbations of human posture during quiet stance. They used eigenvectors of linearized motion equations (called eigenmovements) to analyze the recorded movement and also developed a posture control model using the idea of eigenmovements. Each one of the three eigenmovements was corresponding to the movement mainly done by one of the ankle, knee, and hip joints, respectively. The simulation results stated that using the proposed eigenmovements the controller could control the posture and provide its stability under different conditions using the same strategies used by humans, i.e., ankle and hip strategies. Alexandrov et al. (2005) stated that since the independent eigenmovement control provides an important simplification of control in the multijoint biomechanical system, therefore, it is reasonable to assume that the CNS might use this advantage. Independent eigenmovement feedback control decouples the linearized dynamic equations of the body-skeletal system into a set of independent equations; therefore, the CNS can control each eigenmovement corresponding to the hip, knee, and ankle eigenmovement independently (Alexandrov et al. 2005). In another study, Alexandrov et al. (2001) showed also that the eigenmovements can be independently controlled in a feed-forward manner during voluntary upper trunk bending. Each eigenmovement again corresponded to the movement of one joint. As Nashner and McCollum (1985) and Horak and Nashner (1986) stated, hip and ankle strategies are applied independently under different experimental conditions; this is in complete accordance with the suggestion that the eigenmovements could be controlled independently by the CNS (Alexandrov et al. 2005). Eigenmovements can be considered as "natural synergies" by human body anthropometric properties (Alexandrov et al. 2005). The CNS controls a movement by proper coordination of the eigenmovements. In fact, the CNS gets the information about the current body configuration from the proprioceptive system to estimate the contribution of each eigenmovement (Horak and Macpherson 1996). Therefore, inspired by these facts, as an extension to the idea of the eigenmovements (no linearization applied to the equations of the body-skeletal model), we proposed the agents in the MODEM structure (one might call the agents as generalized eigenmovements). On the other hand, although in the first sight the agents are functioning independently, they indirectly influence each other. Indeed the internal feedback of the efference copy, the movement error signal and the estimated responsibility of each agent modulate the output of each agent (see Fig. 2 for more details). All these facts can be interpreted also as coordinating ability of the CNS.

Certainly, the neurophysiological supports of this idea need to be studied more deeply, while its behavioral support seems to be more evident. However, there are evidences indicating that the neurons selective for arm, leg, and face are located within different parts of the Striatum in the basal ganglia (Crutcher and DeLong 1984a,b); and within each part, there are groups of neurons that respond selectively before and during movement of the corresponding joint, often only in a single direction (Crutcher and DeLong 1984a,b). Similarly, other findings show that there are neurons in other basal ganglia nuclei (subthalamic and globus pallidus nuclei) selective to the direction and speed of individual 
movements (Georgopoulos et al. 1983). These findings led Alexander et al. (1986) to suggest that "the motor circuit may be composed of multiple, parallel sub-circuits, or channels concerned with movement of individual body parts". The structure of the agents of MODEM has been inspired by these observations.

The MODEM architecture suggests also that there are two levels of representation within the sensorimotor system. At the lower level, there exist fundamental experts whose activity is dependent on the accurate motor action but not on the overall task. At the higher level, the "gate selector" block verifies the activations of the lower levels and therefore activity of the higher level is more task-specific and it is not concerned with the precise details of the movement. At the higher level, the activations may form an abstract representation of the task. In addition, some interaction should be seen between modules of an expert. Within an expert, an interaction provided by the feedback of the central command is needed to construct the role of each module in generating the central command of that module (Fig. 2). Many of the recent anatomical and physiological findings pointed out that there are cerebro-cerebellar loops (Haruno et al. 2003; Imamizu et al. 2004). We propose that functions of different parts of the cerebellum correspond to those of parts of the proposed architecture like controllers and predictors. Each part forms a within-layer loop with the cerebral cortex, and these provide the internal cooperation of modules. The interactions between the modules are provided at the cerebro-cortical level by bidirectional cortico-cortical interactions (Haruno et al. 2003). Middleton and Strick (1998) showed that there are closed loop circuits between the cerebellum, basal ganglia, and cerebral cortex. There is some evidence for the cerebellar representation of the lower level controllers supported by neurophysiological experiments in the ventral paraflocculus of the monkey cerebellum during ocular-following responses (Kawato 1999).

Mushiake and Strick (1995) reported that there are neurons in the dentate nucleus related to the context of the task. The experiment accomplished by Mushiake and Strick (1995) was for a sequential task performance and they observed that there were neurons in the dentate nucleus sensitive to the arrangement of the task. The "gate selector" block in our proposed architecture might be the best representation for this observation. Haruno et al. (2003) have stated that basal ganglia loops are involved in sequential movements or switching tasks. The responsibility estimator might also be considered as a suitable representative for this function at the basal ganglia.

In another study, Kermadi and Joseph (1995) stated that cells in the caudate nucleus (one of the nuclei of the basal ganglia) fired during the performance of a sequential task if the task was performed inaccurately. They have concluded that the caudate nucleus plays an important role in error correction during task performance. Therefore, we conclude that there should be parts in the model (auxiliary modules) which are using movement error (see Fig. 2). Consequently, the proposed auxiliary modules might be located in the basal ganglia.

Dove et al. (2000) performed an fMRI study using a task switching study. They recognized regions that were activated for the switching task in the lateral prefrontal cortex as well as in supplementary motor area (SMA), pre-SMA (or F6), lateral pre-motor cortex bilaterally, anterior insula bilaterally, left intraparietal sulcus, and cuneus/precuneus.

Consequently, the mentioned evidences are compatible with the proposed structure for MODEM, which has a hierarchical and distributed architecture, and can be realized by the entire brain network including the cerebral cortex, cerebellum, and basal ganglia.

\section{Conclusion}

In this study, a two-level hierarchical and modularized architecture is proposed to model the human motor control system. The proposed architecture integrates the following concepts: (1) MEX to model the centralized switching between subtasks, (2) Modular autonomous agents to model generalization ability of the CNS in disturbance rejection; the agents contribute in generating the central command based on a distributed switching structure, (3) The notion of the joint compliance characteristics was taken from EPH to enhance the physiological plausibility of the model, and (4) the notion of internal models was also embedded in the MODEM to explain the learning ability of the CNS. The higher-level controller determines the role of each subordinate expert based on prior higher-order knowledge. This block determines which expert should be active in the current situation. Responsibility of each agent is determined by its corresponding estimator block. Responsibilities of the agents can be zero or nonzero and their effects are different in the tracking of each joint angle. In fact, the role of each agent is determined by itself. In other words, in each expert the agents (auxiliary modules) contribute in generating the central command when their function is required. Each agent observes the performance of the main controller and decides to help the main controller or remain silent. This can be interpreted as attention. In other words, the attention of each auxiliary module will be increased if the discrepancy between the prediction of that auxiliary module and the main predictor (movement error) is increased.

We described a possible correspondence between the MODEM structure and the cerebro-cerebellar loop circuits and the basal ganglia. We have also argued that the agents might be considered as generalized eigenmovements. 
Our simulation results confirmed that the proposed architecture is capable of reliable controlling in the presence of additional signal-dependent noise in sensory information, external disturbance, and uncertain environment.

As a consequence of all mentioned facts this architecture seems a more suitable candidate to describe human motor control system than previously developed models.

However, the autonomous selection of the number of experts by the CNS is an important open question. It seems that it requires a sophisticated model of the higher level task understanding. The answer/answers to this question will help us to describe the learning procedure better, and it will also help us to study performance of the MODEM structure for other tasks, like locomotion. In this way one will be able to improve this structure by observing its performance for different tasks and under different conditions.

Acknowledgments Author Mehran Emadi Andani would like to thank the Biologically Inspired Robotics Group (BIRG), EPFL, Switzerland for granting an opportunity to Mehran Emadi Andani to visit their lab and for their scientific collaborations.

\section{References}

Alexander GE, DeLong MR, Strick PL (1986) Parallel organization of functionally segregated circuits linking basal ganglia and cortex. Ann Rev Neurosci 9:357-381

Alexandrov AV, Frolov AA, Massion J (2001) Biomechanical analysis of movement strategies in human forward trunk bending II. Exp Study Biol Cybern 84:435-443

Alexandrov AV, Frolov AA, Horak FB, Carlson-Kuhta P, Park S (2005) Feedback equilibrium control during human standing. Biol Cybern 93:309-322

Balasubramaniam R, Feldman AG (2004) Guiding movements without redundancy problems, In: Jirsa Vk, Kelso JAS (eds) Coordination dynamics. Springer, New York, pp 1-16

Bhushan N, Shadmehr R (1999) Computational nature of human adaptive control during learning of reaching movements in force fields. Biol Cybern 81: 39-60

Blakemore SJ, Wolpert DM, Frith CD (1998) Central cancellation of self produced tickle sensation. Nat Neurosci 1:635-640

Cisek P (2005) Neural representations of motor plans, desired trajectories, and controlled objects. Cogn Process 6:15-24

Craig JJ (1986) Introduction to robotics, mechanics \& control. Addison-Wesley, Reading, MA

Crutcher MD, DeLong MR (1984a) Single cell studies of the primate putamen I. Functional organization. Exp Brain Res 53:233-243

Crutcher MD, DeLong MR (1984b) Single cell studies of the primate putamen. II. Relations to direction of movements and patterns of muscular activity. Exp Brain Res 53:244-258

Darainy M, Malfait N, Gribble PL, Towhidkhoh F, Ostry DJ (2004) Learning to control arm stiffness under static conditions. J Neurophysiol 92:3344-3350

Davidson PR, Wolpert DM (2004) Internal models underlying grasp can be additively 'd. Exp Brain Res 155:334-340

Domen K, Latash ML, Zatsiorsky VM (1999) Reconstruction of equilibrium trajectories during whole-body movements. Biol Cybernet 80:195-204
Dove A, Pollmann S, Schubert T, Wiggins C, Cramon D (2000) Prefrontal cortex activation in task switching: an event-related fMRI study. Cogn Brain Res 9:103-109

Doya K (2002) Multiple model-based reinforcement learning. Neural Comput 14:1347-1369

Doya K, Yoshizawa S (1989) Adaptive neural oscillator using continuous-time back propagation learning. Neural Netw 2:375-386

Doya K, Samejima K, Katagiri K, Kawato M (2002) Multiple modelbased reinforcement learning. Neural Comput 14:1347-1369

Emadi Andani M, Bahrami F, Jabedar Maralani P (2007) A biologically inspired modular structure to control the sit-to-stand transfer of a biped robot. 29th annual international conference of the IEEE EMBS, Cité internationale, Lyon, France, August 23-26, pp 3016-3019

Emadi Andani M, Bahrami F, Jabehdar Maralani P (2009) AMA-MOSAICI: an automatic module assigning hierarchical structure to control human motion based on movement decomposition. Neurocomputing 72(10-12):2310-2318

Farley CT, Houdijk HHP, van Strien C, Lourie M (1998) Mechanism of leg stiffness adjustment for hopping on surfaces of different stiffnesses. J Appl Physiol 85:1044-1055

Feldman A, Latash M (2005) Testing hypotheses and the advancement of science: recent attempts to falsify the equilibrium point hypothesis. Exp Brain Res 161:91-103

Georgopoulos AP, DeLong MR, Crutcher MD (1983) Relation between parameters of step-tracking movements and single cell discharge in the globus pallidus and subthalamic nucleus of the behaving monkey. J Neurosci 3:1586-1598

Gomi H, Kawato M (1993) Recognition of manipulated objects by motor learning with modular architecture networks. Neural Netw 6:485-497

Graybiel AM, Aosaki T, Flaherty AW, Kimura M (1994) The basal ganglia and adaptive motor control. Science 265:1826-1831

Greene PR, McMahon TA (1979) Reflex stiffness of man's antigravity muscles during knee bends while carrying extra weight. J Biomech 12:881-891

Gu X, Ballard D (2006) An equilibrium point based model unifying movement control in humanoids. Robotics: Science and Systems, USA

Guyton AC, Hall JE (2006) Textbook of medical physiology. Elsevier Inc., Amsterdam

Harris CM, Wolpert DM (1998) Signal-dependent noise determines motor planning. Nature 394:780-784

Haruno M, Wolpert DM, Kawato M (2001) MOSAIC model for sensorimotor learning and control. Neural Comput 13:2201-2220

Haruno M, Wolpert DM, Kawato M (2003) Hierarchical MOSAIC for movement generation, vol 1250. International Congress Series, Elsevier Science, Amsterdam, pp 575-590

Hemami H (1982) Some aspects of Euler-Newton equations of motions. Ingenieur-Archiv 52:167-176

Horak FB, Macpherson JM (1996) Postural orientation and equilibrium. In: Rowell LB, Shepherd JT (eds) Handbook of physiology, sect. 12. Oxford University Press, Oxford, pp 255-292

Horak FB, Nashner LM (1986) Central programming of postural movements: adaptation to altered support surface configurations. J Neurophysiol 55:1369-1381

Iacoboni M (2001) Playing tennis with the cerebellum. Nat Neurosci 4:555-556

Imamizu H, Kuroda T, Yoshioka T, Kawato M (2004) Functional magnetic resonance imaging examination of two modular architectures for switching multiple internal models. J Neurosci 24(5): $1173-1181$

Jordan MI, Jacobs RA (1994) Hierarchical mixture of experts and the EM algorithm. Neural Comput 6:181-214

Kandel ER, Schwartz JH, Jessell TM (2000) Principles of neural science. McGraw-Hill Companies 
Kawato M (1999) Internal models for motor control and trajectory planning. Curr Opin Neurobiol 9:718-727

Kermadi I, Joseph JP (1995) Activity in the caudate nucleus of monkey during spatial sequencing. J Neurophysiol 74:911-933

Kim W, Voloshin AS, Johnson SH (1994) Modeling of heel strike transients during running. J Human Movement Sci 13:221-244

Latash ML, Gottlieb GL (1991) Reconstruction of shifting elbow joint compliant characteristics during fast and slow movements. Neuroscience 43((2/3):697-712

Loria A, Lefeber E, Nijmeijer H (2000) Global asymptotic stability of robot manipulators with linear PID and PI2D control. SACTA 3(2):138-149

Miall RC, Reckess GZ, Imamizu H (2001) The cerebellum coordinates eye and hand tracking movements. Nat Neurosci 4:638-644

Middleton FA, Strick PL (1998) The cerebellum: an overview. Trends in Neurosci 21:367-369

Mizrahi J, Susak Z (1982) Elastic and damping response of the human leg to in vivo impact forces. ASME J Biomech Eng 104:63-66

Moraes R, Bahrami F, Patla A (2002) Reprogramming sit-to-stand and sit-to-walk movement sequence under different temporal constraints. The IV world congress on biomechanics, Calgary, Canada

Mushiake H, Strick PL (1995) Pallidal neuron activity during sequential arm movements. J Neurophysiol 74:2754-2758

Nakano E, Imamizu H, Osu R, Uno Y, Gomi H, Yoshioka T, Kawato M (1999) Quantitive examination of internal representations for arm trajectory planning: minimum command torque change model. J Neuro-physiol 81:2140-2155

Nashner LM, McCollum G (1985) The organization of human postural movements: a formal basis è experimental synthesis. Behav Brain Sci 8:135-172
Ozguven H, Berme N (1988) An experimental and analytical study of impact forces during human jumping. J Biomech 21:1061-1066

Partridge LD (1967) Intrinsic feedback factors producing inertial compensation in muscle. Biophys J 7:853-863

Pocock G, Richards CD (1999) Human physiology: the basis of medicine. Oxford University Press, Oxford

Schaal S (2002) Arm and hand movement control. In: Arbib MA (ed) The handbook of brain theory and neural networks, 2nd edn. MIT Press, Cambridge, MA, pp 110-113

Schaal S, Ijspeert A, Billard A (2003) Computational approaches to motor learning by imitation. Philos Trans R Soc Lond B 358: $537-547$

Spagele T, Kistner A, Gollhofer A (1999) Modeling, simulation and optimization of a human vertical jump. ASME J Biomech Eng $32: 521-530$

Todorov E, Jordan M (2002) Optimal feedback control as a theory of motor coordination. Nat Neurosci 5(11):1226-1235

Williams RJ, Zipser D (1989) A learning algorithm for continually running fully recurrent neural networks. Neural Comput 1:270-280

Wolpert DM, Ghahramani Z (2000) Computational principles of movement neuroscience. Nat Neurosci (Suppl) 3:1212-1217

Wolpert DM, Kawato M (1998) Multiple paired forward and inverse models for motor control. Neural Netw 11:1317-1329

Wolpert DM, Miall RC, Kawato M (1998) Internal models in the cerebellum. Trends Cogn Sci 2:338-347

Zatsiorsky VM, Seluyanov V (1983) The mass and inertia characteristics of the main segments of the human body. Biomechanics VIII-B:1152-1159 